\title{
I. Artículos
}

\section{UN MATRIMONIO CONVENIENTE: EL GÉNERO Y LA EDUCACIÓN}

\author{
Sandra Araya Umaña
}

\author{
Recibido 10-III-2003 • Aceptado 13-V-2003
}

\begin{abstract}
Resumen: Desde algunos de los feminismos se han realizado propuestas para la articulación del género en la educación. Dichos planteamientos iluminan la propuesta de equidad de género como un proceso que garantice el acceso de las mujeres y los hombres a un conjunto de saberes, habilidades y destrezas que les permitan estar en mejores y más iguales condiciones de vida.

La educación constituye uno de los vehículos para promover la equidad entre las mujeres y los hombres. Para ello, es imperioso que desde los procesos de formación docente se incorpore un saber dialógico que promueva, en el futuro profesorado, el potencial para la reinterpretación de los contenidos hegemónicos de género y con ello facilitar la introducción de cambios en su práctica laboral y personal.
\end{abstract}

Palabras clave: Feminismos, Equidad de género, Práctica educativa, Formación docente.

\section{Presentación}

En el mundo en general y Costa Rica no es la excepción, el resurgimiento del movimiento feminista en la década del setenta y la realización de la primera Conferencia Mundial de la Mujer en 1975 propician un mayor impulso de las políticas, programas y acciones para el abordaje de la especificidad de las mujeres y la problemática que viven cotidianamente.

Las preocupaciones centrales, en este sentido, conforman un amplio abanico que incluye, entre otras, los derechos políticos y civiles de las mujeres; la igualdad de acceso, de promociones y de salarios dentro del ámbito laboral; su capacitación para el trabajo y proyectos productivos, la importancia de su educación así como la necesidad de garantizar su acceso a los diferentes niveles y opciones educativas; las estrategias para su empoderamiento ${ }^{1}$, la diversidad de las estructuras familiares; la corresponsabilidad de ambos sexos en las tareas de la familia; el hostigamiento sexual y otras formas de violencia contra las mujeres.

En educación, el tema de la igualdad de oportunidades entre mujeres y hombres ha cobrado especial atención por el reconocimiento del papel protagónico que podría desempeñar la institución escolar, si hace suyo el abordaje sistemático y sostenido de las anteriores preocupaciones. 
Como producto de ello, se concibe una escuela con capacidad de resignificar los contenidos hegemónicos de género, es decir, las desigualdades sociales que se producen a partir de la construcción cultural y así contribuir con el establecimiento de una sociedad más justa y democrática.

Para la consecución de lo anterior, los diversos feminismos han planteado propuestas para el abordaje de la igualdad de oportunidades en la educación. Estas propuestas se originan en los países anglosajones y por las particularidades de los países latinoamericanos no es conveniente traslapar en forma automática sus respectivas experiencias, no obstante, sus postulados iluminan el planteamiento de la equidad de género que propongo en este texto y de ahí su particular exposición. Veámoslo.

\section{Género y educación}

El abordaje sistemático de lo que les ocurre a las mujeres en el sistema educativo se inicia en la década del 70, fundamentalmente en Estados Unidos y en Europa, por medio del encuentro entre el pensamiento feminista y las formulaciones derivadas de la sociología de la educación.

En efecto, desde la sociología de la educación y desde los años cincuenta se empieza a perfilar una tendencia que cuestiona hasta qué punto el sistema educativo era, como pretendía ser, un espacio creador de igualdad de oportunidades. Los aportes de Berstein (1988) en Inglaterra y Bourdieu (1984) en Francia abren una nueva visión sobre la escuela que posibilita analizar el sistema educativo como un espacio investido por el poder, carente de neutralidad y, por lo tanto, creador y legitimador de identidades sociales jerarquizadas. Se plantea, por consiguiente, la necesidad de una educación emancipadora y liberadora comprometida con la superación de las desigualdades sociales.
Para comprender el impacto del sistema educativo sobre los individuos singulares no bastaba con un discurso general: había que analizar cómo se establece la interacción, en forma muy concreta. Y, por lo tanto incorporar el análisis microsociológico, la consideración de lo que ocurre en la escuela y en el aula. Algunos conceptos fueron fundamentales para ello, como toda la aportación foucaultiana relativa a la microfísica del poder. La escuela, el aula, la interacción cotidiana, permitieron descubrir que el sistema educativo es mucho más que un expendedor de títulos, es un espacio de socialización diferenciada, en el que hay reglas sumamente estrictas, aunque invisibles que moldean con gran precisión las personalidades individuales, que construyen el éxito y el fracaso, que separan a quienes están destinados a tener responsabilidades y a tomar decisiones, de quienes están destinados a plegarse a ellas (Subirats, 1999, p. 21-22).

No obstante lo anterior, los análisis de los sesenta y de los setenta provenientes de la sociología de la educación, y en particular de la pedagogía crítica, no trataron el tema de las mujeres como un foco de sus alternativas de liberación. Fue la distinción teórica entre sexo y género, introducida en la década del 70 por los estudios feministas, la que colocó las particularidades de lo femenino en el sistema educativo e inició con ello un nuevo debate acerca del papel que cumplía la educación en la perpetuación y reproducción de las desigualdades entre mujeres y hombres.

Es en Francia, Italia, Inglaterra, España y Estados Unidos en donde se comienzan a realizar análisis sobre los diversos aspectos de la realidad escolar y su forma de incidencia en la socialización de las niñas (Subirats, 1999).

Si bien quienes escriben sobre pedagogía feminista no suelen situar sus trabajos ni sus escritos en marcos específicos de pensamiento feminista, los estudios que surgen presentan diferentes énfasis y orientaciones según las líneas de los feminismos a los que se adscribieron $\mathrm{y}$, aunque es difícil mapear todos los feminismos existentes (Belausteguigoitia y Mingo, 1999), es posible agrupar cuatro tendencias dentro de las prácticas feministas anglosajonas y, de esta manera, exponer su 
vinculación con la educación: el feminismo radical, el feminismo liberal, el feminismo socialista y, en fechas más recientes, el feminismo postestructuralista ${ }^{2}$.

\section{El feminismo radical}

Las prácticas del feminismo radical conciben como el fundamento de la opresión a las estructuras de dominación masculina y patriarcal. En este marco el concepto de patriarcado adquiere relevancia como productor de sentidos masculinos y femeninos, pero centrado en el sistema de significaciones masculinas. No obstante, y a pesar de sus aportes, como concepto teórico el patriarcado fue criticado, en particular por las feministas socialistas, por el carácter omniexplicativo que adquirió.

Las prácticas del feminismo radical no buscan la igualdad de oportunidades entre los sexos, sino la abolición de la dominación masculina y sus formas de limitación y definición del conocimiento. En este sentido, la meta de la educación feminista no es la igualdad en el conocimiento, el poder y la riqueza, sino la abolición del género como realidad cultural opresiva (O'Brien cfr. Acker, 1995).

En educación, la producción escrita en esta línea presenta dos tendencias fundamentales: 1) el monopolio de la cultura y del conocimiento por parte de los hombres y 2) las normas establecidas para los sexos en la vida cotidiana de las escuelas.

Un aspecto no abordado dentro de esta perspectiva es el que se refiere a cómo reeducar a los niños y a los hombres para la superación de las relaciones asimétricas.

El feminismo radical ha tenido gran auge en Italia y en Inglaterra.

\section{El feminismo liberal}

La meta fundamental del feminismo liberal es asegurar la igualdad de oportunidades de los sexos. En sus prácticas existen tres temas esenciales: 1) igualdad de oportunidades, 2) socialización y estereotipos de sexo y 3) discriminación sexual (Acker, op. cit.). Es por ello que en educación han puesto su atención en: la coeducación; los patrones de socialización de niños y niñas en la escuela y los límites que estos imponen al desarrollo de ellas; el análisis de estereotipos sexuales en los libros de texto; el estudio de las diferencias que se dan en las actitudes, los comportamientos y lenguaje de los docentes y las docentes hacia niños y niñas en el aula y la promoción y potenciación de las mujeres para su ingreso y permanencia en carreras consideradas masculinas.

Los cuestionamientos a estas prácticas apuntan al carácter acrítico de la incorporación igualitaria de las mujeres a la educación y al mercado laboral, así como a la ausencia de análisis de la dinámica del modelo capitalista de producción y sus consecuencias para las mujeres. Por ello, son tildadas de "ingenuas" y "meritocráticas". Sin embargo, su mayor fuente de crítica proviene del esencialismo que las caracteriza, pues ubican a las mujeres como si fueran un bloque monolítico, sin distingo de clase, color, opción sexual y etnia, entre otros.

Estas prácticas se han desarrollado fundamentalmente en España, Inglaterra, Estados Unidos y Australia y son las que han tenido mayor eco en América Latina.

\section{El feminismo socialista}

El feminismo socialista contribuyó en parte a la batalla contra las invenciones esencialistas, pero aportando una nueva veta acerca de los orígenes de la inequidad: "el modelo económico sostenido a partir del capitalismo es la causa fundamental de la opresión femenina, pues se beneficia de ella". Así, según sus planteamientos, al eliminar el modelo económico centrado en la acumulación desigual del capital, otros tipos de sometimiento y opresión desaparecerían. 
Las prácticas derivadas de esta corriente han sido influidas fuertemente por las tendencias neomarxistas dentro de la sociología de la educación. Un concepto fundamental usado dentro de los escritos producidos en esta línea es el de reproducción. En este sentido, han puesto especial interés en aclarar la forma en que el género y las relaciones de poder clasistas son reproducidas continuamente en el medio escolar; en el papel de la escuela en la formación de hombres y de mujeres de clase trabajadora y en su perpetuación como tales; así como en la relación que guardan la familia, la escuela y el mercado laboral con el mantenimiento de las relaciones de dominio entre géneros y clases sociales. Se han especializado en estudios que evidencian las clasificaciones de las profesiones, empleos, y trabajos femeninos como menores, peor pagados, fuera del campo de la tecnología, la computación y los puestos de dirección.

Según Acker (op. cit.) y Gore (1996), gran parte del trabajo feminista socialista en educación consiste en la formulación de argumentos teóricos, en investigaciones históricas o en análisis políticos. Comparativamente hay pocos estudios empíricos sobre los procesos en la escuela, por lo que desde este feminismo es incipiente el diseño de prácticas educativas dirigidas al salón de clases.

Críticas provenientes de las mujeres negras contribuyeron a que el feminismo socialista tomara conciencia de la intersección de factores como el género, la clase y la sexualidad en el modelaje de las niñas y de las mujeres tanto dentro como fuera del aula escolar.

En forma paralela, en la sociología de la educación, han surgido estudios que han tratado de dar mayor peso a la forma en que las personas y los grupos en general se resisten a las ideologías dominantes y a los procesos de control social (Giroux, 1985). Ambos aspectos interactuaron para la aparición de los estudios culturales dentro del feminismo socialista, los cuales han transitado del análisis de las formas hegemónicas de dominación a la capacidad, las estrategias y las alternativas de las personas para resistirse al control social. De esta manera, contienen una base neomarxista muy sólida, además de considerar otros aspectos en el análisis de las condiciones de opresión de las mujeres.

\section{El feminismo postestructuralista y la pedagogía crítica}

En Estados Unidos, durante la década de los 80 crece el interés por estudiar las teorías que estudiaban la emancipación y la liberación de grupos marginales. Es así como las teorías de autores como Freire -de circulación en América Latina desde la década del 70- consolidan en el campo educativo la aparición de la pedagogía crítica, cuyos principales exponentes son Henry Giroux, Peter Mclaren y Donaldo Macedo ${ }^{3}$.

El nacimiento de la pedagogía crítica introdujo un nuevo marco de reflexión y análisis teórico que rompía con la forma tradicional de enfocar el pensamiento educativo, aunque como se anotó supra, el género no era objeto de análisis del impacto del poder o del discurso de la opresión.

En este contexto, la construcción teórica del feminismo postestructuralista acentúa el análisis de la interrelación de los diferentes vectores que construyen la diferencia, privilegiando un vector $u$ otro (el género, la raza, la orientación sexual, entre otras) según sea el contexto. Desde sus filas, la pedagogía crítica ha sido fuertemente criticada (Gore, 1996, Ellsworth, 1999; Lather, 1999; Orner, 1999) no solo por la ausencia del tratamiento de la diferencia en sus diversas expresiones, sino también por su universalidad y utopía dado el carácter sumamente abstracto del lenguaje que utiliza.

Estos cuestionamientos han producido textos (McLaren, 1997; Apple, 1997) que intentan articular las diferencias de 
género y de orientación sexual dentro del discurso de la pedagogía crítica ${ }^{4}$. No obstante, Freire y Macedo (cfr. Belausteguigoitia y Mingo op. cit.) señalan que es imposible estructurar una pedagogía que combata todas las formas de opresión a las que están sometidas las personas por lo que se hace necesario que cada forma particular de opresión derivada de factores tales como la clase social, el género, la raza, la sexualidad, la edad, sea abordada por pedagogías individuales que ataquen uno a uno dichos factores o bien a un binomio acoplado de éstas.

La discusión no está agotada, en particular entre las feministas postestructuralistas estadounidenses, inglesas o australianas. Sin embargo, para el caso de América Latina es necesario contextualizar esta discusión.

Efectivamente, según mi criterio, las críticas a esta pedagogía así como las posturas del feminismo postestructuralista deben tomarse con cautela, debido a la especificidad de las realidades sociales latinoamericanas y, más particularmente, las educativas. A ello hay que sumar la inclusión de esta pedagogía -aunque con un carácter marginal (Gore op.cit.)- en los programas de formación docente de aquellos países, lo cual expresa una notable diferencia con respecto a las experiencias pedagógicas formativas de los países latinoamericanos en general y de Costa Rica, en particular.

Por otra parte, los proyectos que apoya la pedagogía crítica, que se oponen a las posiciones opresoras de clase social, han abierto las posibilidades de cuestionar el sistema educativo y de postular su ineludible compromiso con las vindicaciones de los grupos marginales. Si en un inicio, sus planteamientos obviaron el género o si en la actualidad lo incluyen bajo la técnica de adhesión, esto no la inhabilita como herramienta teórica para una mayor comprensión de las relaciones de desigualdad entre los sexos. A ello hay que agregar el riesgo que conllevan los planteamientos feministas postestructuralistas por su escisión meticulosa de las diferencias, lo que dificulta aún más las acciones de movilización de los grupos sociales.

No obstante, sus planteamientos, así como los de los demás feminismos, han colocado en la agenda de discusión un punto de encuentro: ya no se trata de describir diferencias de comportamiento entre niñas y niños, sino de saber qué se puede hacer para que la escuela deje de ser sexista y discriminatoria para las mujeres y por ende sea un espacio promotor de la equidad entre los sexos.

\section{La equidad de género: propuestas educativas}

El modelo dominante en el sistema educativo actual es el de escuela mixta. Este modelo establece estatutariamente el principio democrático de igualdad para todas las personas y defiende la educación conjunta para mujeres y hombres como un compromiso básico del sistema educativo. En consecuencia, la provisión de educación tiene que ser la misma para todas las personas en el ámbito curricular y pedagógico.

Este modelo no reconoce las desigualdades entre los hombres y las mujeres, pues el principio del que parte es el de la "homogenización" de la enseñanza. En consecuencia, el género no es una variable relevante para el trabajo escolar. El reconocimiento de las diferencias entre los sexos es entendido, a menudo, como sinónimo de discriminación, pues se parte de que la institución escolar es democrática y justa (Bonal, 1997:39).

Por lo anterior, la escuela mixta no ha logrado generar condiciones que aseguren la igualdad entre mujeres y hombres, pues el modelo pedagógico dominante tiene un carácter androcéntrico: Ha sido construido teniendo en cuenta únicamente las necesidades culturales dominantes en la actividad pública y concede, en forma 
oculta, una atención diversa a los hombres y a las mujeres y con ello reproduce las pautas culturales que asignan mayor valor a lo masculino en detrimento de lo femenino.

La posición de igualdad de oportunidades se postula en educación como una alternativa que contrarresta los efectos de la escuela mixta. Desde esta posición se plantea como meta educativa la coeducación, o sea, una escuela con objetivos orientados a la modificación radical de las relaciones interpersonales.

Es en España, donde esta posición tiene una fuerte trayectoria. En países como Reino Unido y Dinamarca (Acker, op. cit.; Mette, 1992), la igualdad de oportunidades ha seguido una trayectoria similar, aunque con las particularidades provenientes del abanico de feminismos existentes y la estructura social de cada uno de los respectivos países.

Para el logro de la equidad de género en la educación, la posición de igualdad de oportunidades se centra en la noción de derechos igualitarios para mujeres y hombres sustentando que ello se consigue sólo en el momento en que las mujeres puedan acceder a los espacios normalmente ocupados por los hombres.

La igualdad de oportunidades ha sido fuertemente criticada por grupos feministas de corte radical por considerar que ofrece una perspectiva que se adecua a las necesidades de los políticos. Particularmente, en la medida en que establece estrategias viables y objetivos aparentemente factibles con los recursos existentes y porque el análisis que sirve de apoyo a estas iniciativas tiende a centrar los problemas en las mujeres (Arnot, 1992). De estos grupos surge la posición de eliminación del sexismo.

No obstante que presenta en su interior diferentes ramificaciones -provenientes de los feminismos existentes- el grupo que postula la bandera de la eliminación del sexismo tiene en común su interés por la transformación radical del sistema educativo modificando su base de poder (Weiner y Arnot cfr. Arnot, op. cit.).

Desde esta posición el problema de las mujeres tiene que ver menos con sus elecciones y más con su opresión ante la dominación masculina en las escuelas. El sistema educativo se considera enmarcado por los intereses y la cultura masculina y por consiguiente como generador del silencio y la marginación de las profesoras y las alumnas en las escuelas. Promueve, por tanto, una reflexión crítica sobre las estructuras de poder que sustentan y perpetúan las diferencias y discriminaciones, y alerta sobre el peligro de integrar a la mujer a un mundo masculino, sin cuestionarlo y modificarlo previamente (Arnot, op. cit.).

La diferencia fundamental entre ambas posiciones es la concepción de justicia social y la forma de lograrla. Para la posición de igualdad de oportunidades la justicia social se basa en la libertad individual y se logra mediante la igualdad de acceso a la educación. En la posición eliminación del sexismo, la justicia social es equivalente a la eliminación de las barreras estructurales y se lograría tomando medidas para asegurar la igualdad de resultados (Bonal, op. cit.).

En este debate, especial atención merece la pedagogía de la diferencia sexual como una nueva perspectiva feminista radical sobre la educación. Esta perspectiva surgió en Italia a inicios de los ochenta por obra de la Librería de Mujeres de Milán y posteriormente de la Comunidad Filosófica Femenina Diótima de la Universidad de Verona. Consiste, esencialmente, en cambiar la sociedad de modo que en ella tenga curso lo que una mujer es y quiere ser, es decir, consiste en la creación de la subjetividad femenina; en la introducción de lo femenino a lo simbólico; en traer al mundo el mundo femenino (Diótima, 1996; Librería de Mujeres de Milán, 1987). 
La política de la diferencia sexual no asume ya como referencia central la discriminación y la injusticia social que sufren las mujeres y en consecuencia ha desplazado su interés desde la lucha de la reivindicación de mayores derechos e igualdad de oportunidades para nuestro sexo, hacia la afirmación de una existencia social libre de las mujeres en un mundo común... De tal modo se ha trastocado el esquema clásico de cierto feminismo que considera esencial la relación hombre/mujer con la finalidad de hacerla más justa y mejor, concibiendo las relaciones entre mujeres como el medio para llegar a ese objetivo (Piusi, 1999a, p. 277-278).

Efectivamente, una de las características de la pedagogía de la diferencia es que presenta una línea de pensamiento orientada al redescubrimiento y fortalecimiento de las relaciones entre las mujeres como una forma de combatir las estructuras patriarcales.

Desde esta posición, las mujeres no deben buscar igualarse a los hombres, sino más bien exaltar su condición femenina para combatir cualquier forma de opresión sexista desde la libertad femenina, la cual se conquista y se finca en las relaciones sociales con otras mujeres. Retoman el término affidamento (sin traducción en castellano) para denominar las relaciones de fe, fidelidad, confianza y solidaridad entre las mujeres. Para ellas, la política de la diferencia sexual no viene después de alcanzar la igualdad entre los sexos, sino que más bien la sustituye.

En Italia y desde hace algunos años, profesoras de escuelas elementales y de secundaria, educan en la diferencia, marcando con un signo sexuado el signo de la libertad femenina, provocando transformaciones significativas mediante el desplazamiento de la autoridad y de la proyección sobre su sexo (actividades educativas con grupos homogéneos por sexo, aplicación de unidades didácticas para las mujeres, cursos de formación y actualización para las profesoras, sexuación de la lengua y de los contenidos de los programas) (Piussi, 1996; 1999b).

Este discurso, junto con el que proviene de la posición de eliminación del sexismo, no han tenido ningún impacto en América Latina.
Es el discurso de igualdad de oportunidades inscrito en el feminismo liberal el que se ha propiciado desde los sistemas educativos latinoamericanos. Argentina y Chile son los países que al respecto han generado mayores propuestas. En ambos países el género se ha asumido como uno de los aspectos centrales de la política educativa, con dos rasgos importantes: es planteado como parte de la educación en derechos humanos; es formulado como coeducación y se postula con dos dimensiones: "igualdad de oportunidades" y "eliminación del sexismo" (Tovar, 1997) ${ }^{6}$.

\section{Equidad: Una aproximación conceptual}

La posición de igualdad de oportunidades está iluminada por el feminismo de la igualdad, de raíz ilustrada el cual, a su vez, se articula en torno a las ideas de igualdad y libertad. Por su parte, la eliminación del sexismo se ancla en el feminismo de la diferencia, en el que la noción de igualdad pasa por incorporar la idea de diferencia, es decir, que las mujeres no sólo adquieran posiciones de poder, sino que puedan hacerlo a su modo.

A pesar de que ambas posiciones se critican recíprocamente es posible construir, a partir de ellas, el concepto de equidad con una dimensión que trascienda a la igualdad.

Efectivamente, y de acuerdo con la posición de eliminación del sexismo, la igualdad de oportunidades no resuelve el problema de cómo integrar los valores considerados tradicionalmente femeninos y que de hecho continúan subsistiendo, pero interiorizados solo por las niñas y las mujeres. Ello conduce -sin el esencialismo ${ }^{7}$ de algunos feminismos de la diferencia- al reconocimiento de la diferencia de un modo éticamente desafiante y políticamente transformador para así crear las condiciones que coadyuven a la superación de la dominación y la desigualdad entre los sexos. 
Para lograr la equidad no basta con que las mujeres puedan acceder en igualdad de condiciones a los ámbitos tradicionalmente considerados masculinos. Se requiere la transformación del conjunto de normas sociales y la transformación del género, hasta su desaparición, por medio de la fusión de los valores, de las actitudes y de las prácticas históricamente atribuibles a las mujeres y a los hombres.

Lo anterior equivaldría no a la supresión de la diferencia, sino más bien a que ésta -sea de sexo, cultura, de raza, color, de lengua, de religión- deje de ser la base sobre la que se funda y legitima una jerarquía de poder y así, de esta forma, pasar al reconocimiento de los rasgos constitutivos de la diferencia como rasgos que presentan un gran valor para la vida de la colectividad, portadores de un principio de renovación o renegociación social que los hace deseables por sí mismos (Subirats, 1998).

No se trata tampoco de la mal llamada cultura de la tolerancia, pues, aunque en determinados momentos la tolerancia puede ser positiva, el término sugiere todavía una jerarquía en la que, quien tiene poder para ello acepta convivir con lo distinto, en la forma de una concesión o de un acto de magnanimidad.

Así concebida, la equidad significa el proceso que garantiza el acceso de las mujeres y los hombres a un conjunto de saberes, habilidades y destrezas que les permiten estar en mejores y más iguales condiciones de vida. Significa educar en la diferencia y desde la diferencia para validar las características humanas (ternura, deseo, solidaridad y raciocinio-lógico, entre otras) como expresiones del mismo sustrato, no exclusivas de ninguno de los sexos y necesarias para la supervivencia humana, en general.
Lo deseable es la construcción de una nueva forma de vida que permita establecer nuevos equilibrios entre lo público y lo privado, el trabajo productivo y el reproductivo. Pero estos nuevos equilibrios no deben estar basados en un regreso a partir en dos el universo social, y en atribuir una mitad a cada uno de los sexos, sino en que todos los individuos, hombres y mujeres, contribuyan equilibradamente a ambos aspectos de la vida. Es decir, al establecimiento de un nuevo pacto, o un nuevo contrato, como se ha dicho a menudo, entre hombres y mujeres, para una partición del trabajo socialmente necesario que no tenga el carácter de la división sexual del trabajo, sino de la asunción individual de parcelas situadas en ambas esferas (Subirats, 1998, p. 61).

Es posible que la consecución de lo anterior requiera de la asunción de una serie de medidas transitorias que, en primer término, promuevan que las mujeres se reconozcan a sí mismas como sujetas de derecho y con condiciones para el desarrollo de un proyecto de vida autónomo. El affidamento, en este sentido, es uno de los elementos más atractivos de la propuesta de la pedagogía de la diferencia sexual pues, ciertamente, es necesario generar condiciones para que las mujeres se (re) descubran como parte de un mismo grupo que, por la intervención de mecanismos patriarcales, se ve a sí mismo de manera fracturado. Esto, sin embargo, y especialmente para el caso de los países de América Latina, debe comportar un análisis de los significados, no solo de tener un cuerpo de mujer, sino también de tener un cuerpo negro o un cuerpo indígena que, a su vez, están asociados a una clase social.

Lo anterior conduce al segundo componente de la equidad: la valoración social de las diferencias como una forma de asegurar y perfeccionar la justicia. Es decir, como medio para asegurar que cada cual reciba lo que requiere para el logro de su respectiva autonomía. Este componente refiere a la dimensión ética de la equidad, pues lo que se busca promover es la verdadera igualdad como sinónimo de la justicia social. 
En efecto, y de acuerdo con el planteamiento aristotélico, la igualdad debe ser para los iguales y la desigualdad para los desiguales (Libro V de la Ética Nicomaquea), pues como el estagirita lo advierte:

Si las personas no son iguales, no tendrán cosas iguales. De aquí los pleitos y las reclamaciones cuando los iguales tienen y reciben porciones no iguales, o los no iguales porciones iguales... Lo justo es algo proporcional (Aristóteles, 1967, p. 61-63).

Así, la equidad sitúa a las personas en sus dimensiones originales, sin realizar falsas abstracciones de igualdad, que las distancian de su historia personal, social y cultural. Es decir, somos iguales entre nosotros (y nosotras), solo porque somos diferentes (Touraine, 1998, p. 63).

Las mujeres y los hombres, por consiguiente, son bloques diferenciados tanto por las condiciones de género como por la edad, la clase social, la raza, la opción sexual y la religión. Estos factores interactúan entre las mismas mujeres y entre los mismos hombres, generando también diferencias en el interior de ambos grupos, las cuales, para efectos del abordaje de la equidad, deben ser considerados como factores relevantes de las diversas expresiones del género.

\section{El compromiso educativo}

La equidad entre los sexos debe ser una meta educativa. Por su condición de instancia pública y el ámbito de captación de una población, que por su ciclo de vida (niñez, y adolescencia) es más permeable al conocimiento y a la innovación (Fischel, 1994), la educación puede contribuir al diseño y desarrollo de diferentes estrategias que permitan la consecución de la equidad. No se trata de hacer de ella el motor del cambio social, pues como microcosmos que es, constantemente se tiene que enfrentar con las fuerzas que devienen del medio social. Sin embargo, por su contenido político ideológico (Sobrino, 1987), sí puede ser un campo fértil para el cuestionamiento y para la cooperación en la transformación de las relaciones entre las mujeres y los hombres.

Si bien es cierto el sistema educativo no puede eliminar las desigualdades individuales cuando están insertas en el conjunto social, también es cierto que no hay cambio social si no empieza a producirse en algún punto de la sociedad y que todo cambio habido en una parte del sistema repercutirá en las otras partes (Subirats,1994: 73).

Para lo anterior es imperativo considerar la práctica educativa y los factores que intervienen en ella. Es indiscutible que dentro de éstos, el papel del estudiantado como actor protagónico ocupa un lugar preponderante pero, también es incuestionable que la actividad del estudiantado requiere de una guía experta y de un medio ambiente estimulante que sólo el educador o la educadora y la institución educativa pueden ofrecer (Tedesco, 1998).

En este sentido, debe tomarse en consideración que el término educadora o educador designa en forma genérica una función social cuya misión fundamental es la de enseñar, la cual, a su vez, se distingue como un acto relacional entre dos o más personas con un carácter intencional dirigido siempre a facilitar la construcción de los aprendizajes (Fenstermacher, 1990).

Sin embargo, por las particularidades biológicas y biográficas del profesorado y del alumnado, así como por el entorno cultural y las características del contexto social en que se inserta la relación educativa, la enseñanza tiene un carácter eminentemente social, configurando, por lo tanto, una red de relaciones interpersonales que están mediatizadas por las características personales del profesorado y del alumnado, sus cosmovisiones particulares, sus expectativas, el currículo explícito y el oculto, los materiales que se utilizan, la configuración de los espacios, las relaciones afectivas que se establecen, los ritmos de aprendizaje y por las estrategias de enseñanza que utiliza el profesorado (García y Canal, 1995). 
Por ello, quien enseña remite a personas de una raza, de una clase social y un sexo específicos, cuyas biografías están íntimamente ligadas a la trayectoria económica, política e ideológica de sus familias y comunidades; a las economías políticas de sus vecindarios y a las relaciones de desigualdad de la sociedad en general. Ello hace que en el acto relacional el papel del profesorado no sea neutral debido a que su acción está mediatizada por lo que cree, lo que dice y lo que hace (Apple, 1997, Postic, 1996).

...en toda determinación curricular hay un posicionamiento educativo, hay una intencionalidad actitudinal, una idea subyacente sobre el individuo que se pretende formar, una mimesis personal o social que se trata de extender y reproducir por medio de la actividad de enseñar. Por todo ello la escuela no es una institución en la que se produce de manera aséptica y neutral transmisión de información sobre conocimientos culturales y académicos (Hernández y Sancho, 1993, p. 102).

En virtud de lo anterior, el profesorado ejerce una forma de poder, una fuerza social que puede actuar a favor del cambio o de la continuidad social (Carr, 1996).

En este sentido, la práctica educativa es más que una actividad; es una construcción social que expresa supuestos ideológicos controvertidos, concepciones sociales, educativas y epistemológicas, al lado de una multitud de creencias subjetivas, provocando que el cambio desde la educación adquiera inevitablemente una dimensión de complejidad.

Los cambios de prácticas educativas son lentos porque exigen cambios de contenidos, de estrategias metodológicas y ello reclama conversiones a nivel subjetivo, discursos teóricos apropiados y mejores condiciones materiales ya que la innovación pedagógica es un problema de cambio de cultura [el resaltado no es del original] (Gimeno, 1989, p. 18).

La práctica educativa como práctica reflexiva ${ }^{8}$ es un elemento clave para propiciar el cambio en educación que conlleve la equidad en las relaciones entre las mujeres y los hombres. Ello significa que dicha práctica sería el motor del cambio lo cual induciría, por un lado, la emancipación del profesorado como personas constructoras de su propio conocimiento y de su acción, y por el otro, la posibilidad de convertir la educación en un medio para la ilustración individual y para la acción social colectiva, conectada profundamente con los ideales emancipadores de la racionalidad, la justicia y la libertad (Carr, op. cit.).

En esta perspectiva, el proceso de formación docente cobra vital importancia, pues desde él se pueden construir las condiciones para que el profesorado se constituya en agente multiplicador de la equidad entre los sexos. Ello supone una relación dialéctica entre el cambio cultural y las prácticas educativas en la que el primero, no solo provenga del exterior y actué sobre las segundas sino que, estas prácticas además de propiciar el cambio, serían consolidadas, a su vez, por él.

\section{La formación de educadoras y educadores}

El concepto de formación adquiere distintos significados según el énfasis que, entre otros, se le asigne a los programas, a las instituciones formativas y a los objetivos (Ferry, 1990, 1997; Filloux, 1996).

Así, el concepto de formación, al igual que muchos otros, es susceptible de múltiples enfoques.

La noción de formación, sin embargo, adquiere sentido cuando señala una acción reflexiva que permite pensar sobre lo que se hace, sobre su significación, sobre los fracasos y los éxitos que se viven y sobre la dinámica personal y profesional, en general.

La formación es, por tanto, un proceso de desarrollo individual tendiente a adquirir o perfeccionar las capacidades 
de sentir, de actuar, de imaginar, de comprender y, de aprender (Ferry, 1990).

A partir de los anteriores enunciados se puede afirmar que una formación no se recibe. Nadie puede formar a otro $u$ a otra, pues son las personas las que se forman y se desarrollan. No obstante, si bien cada cual se forma a sí mismo y a sí misma, también es cierto que las personas se forman solo por mediaciones (Ferry, 1997).

Las mediaciones son diversas: las experiencias vividas, la relación con las otras y los otros, las lecturas, las circunstancias, los contenidos de aprendizaje, el currículo y el propio profesorado.

En efecto, el modelo pedagógico que adopten las formadoras y los formadores tiende a imponerse como modelo de referencia del estudiantado -en este caso las futuras y los futuros docentes-, quienes se ven condicionadas y condicionados a reproducir los procedimientos, las actitudes, el estilo de comportamiento desarrollado por las formadoras, los formadores y por la institución de formación.

Los efectos de estructuración y de impregnación producidos por el dispositivo de formación pueden llegar a ser más fuertes que sus discursos. Es la razón por la cual el curso magistral practicado en la universidad, al cual se le agrega el prestigio de un saber innegable y de la autoridad que confiere, queda como el prototipo de la actividad enseñante, a pesar de todo lo que se ha escrito y dicho en alabanza de los métodos activos (Ferry, 1990, p. 61).

Por lo anterior, se espera que todo proceso de formación sea innovador y se articule y dé respuesta a las nuevas demandas de la sociedad.

En la formación inicial debería producirse una primera transformación y eventuales cambios en las actitudes, valores y funciones que el futuro o la futura educadora atribuye a la profesión docente y se debería impulsar la adopción de determinados hábitos que influirán en el ejercicio posterior de la enseñanza.
Lo que se espera de todo proceso formativo innovador es que contribuya a forjar, en los que participan en él, un conjunto de capacidades, destrezas y actitudes con sus correspondientes fundamentos axiológicos que les permitan alternar, recrear y reconvertir continua y lúcidamente sus habilidades y competencias, según lo exijan los requerimientos de los contextos culturales, sociales y escolares en que les toque ejercer su quehacer, en que tienen lugar sus prácticas (Cornejo, 1999 , p. 60).

La acción formativa, actividad formativa $o$ acciones de formación corresponde, entonces, a un conjunto de conductas, de encuentros e interacciones entre las personas involucradas que puede perseguir múltiples finalidades, explicitadas o no, y por las cuales hay una intencionalidad de cambio. O sea, el problema del cambio es el problema fundamental (o debería ser) de la formación docente (Marcelo, 1994).

Para lograr el cambio en la educación, es preciso que, en primer término, en los procesos de formación docente se haga un reconocimiento del valor de las desigualdades sociales procedentes de la construcción cultural del género. No es el currículo común ni la metodología homogénea la que garantizará la igualdad de oportunidades para las mujeres y los hombres sino, la atención a las diferencias individuales mediante una metodología y un currículo lo suficientemente flexibles y diversificados como para potenciar el intercambio y la participación activa del estudiantado en los procesos de aprendizaje, experimentación y comunicación (Gimeno y Pérez, 1997).

La realización plena de lo anterior constituye un ideal que servirá de correctivo a la excesiva estandarización de la enseñanza que no ha considerado las características particulares que la cultura ha conferido a las relaciones entre mujeres y hombres.

El proceso de formación del profesorado puede constituirse en la plataforma en donde inicie la concreción de este ideal. 


\section{Notas}

1. Los estudios de la mujer han buscado un equivalente en castellano del verbo empower y del sustantivo empowerment. Las revisiones realizadas señalan que no hay nada inmanente en estos términos. En español significan "dar poder" y "conceder a alguien el ejercicio del poder” (León, 1997).

2. Es necesario insistir que las autoras que construyen la pedagogía feminista no mencionan unas líneas de pensamiento referidas a la "pedagogía feminista liberal", la "pedagogía feminista radical", o a la "pedagogía feminista socialista". Tampoco es posible imponer tales categorías a los trabajos publicados, en los cuales, es observable la coexistencia de distintas tradiciones de pensamiento feminista aun dentro de un mismo texto.

3. Según Gore (1996) en la pedagogía crítica es posible distinguir dos líneas de pensamiento. Una que hace hincapié en la articulación de una amplia visión social y educativa y la otra, que muestra una mayor preocupación por el desarrollo de prácticas docentes explícitas adaptadas a contextos específicos. Proponentes claves de la primera son Henry Giroux y Peter McLaren y Paulo Freire e Ira Shor de la segunda.

4. Las teóricas del feminismo postestructuralista estadounidense son profundamente escépticas con relación a estos discursos dado que el género podría ser solamente un concepto sumado en ellos. (Para ampliar véase Belausteguigoitia y Mingo, op. cit).

5. Por supuesto que al igual que con otras teorías sociales es necesario analizar la pedagogía crítica con bisturí feminista para detectar, relevar y combatir sus rasgos sexistas.

6. En Argentina se creó en 1991 el Programa Nacional de la Promoción de Igualdad de Oportunidades para la Mujer en el Área Educativa a cargo del Ministerio de Cultura y Educación. En Chile hay una Propuesta $\mathrm{Cu}-$ rricular Ministerial en la que el tema género está presente en uno de los seis contenidos estipulados así como en los objetivos fundamentales en los que el respeto a la equidad entre los sexos se plantea como un valor a ser internalizado, como un deber ser, un sentido, un acto voluntario (Tovar, 1997).
7. El esencialismo se caracteriza por sustentar que existen esferas psíquicas, sociales y culturales separadas para mujeres y hombres. En esta diferenciación, las posturas esencialistas glorifican y ensalzan a las mujeres por el hecho de serlo y por la consideración de que son más fuertes, más capaces y más inteligentes. Representa una versión feminista del eterno femenino.

8. La práctica reflexiva es un movimiento internacional que se ha desarrollado en la enseñanza y en la formación bajo el lema de la reflexión, y aporta como novedoso el reconocimiento de que la producción de conocimiento no es propiedad exclusiva de los centros universitarios, sino que el profesorado tiene también teorías que pueden contribuir a la constitución de una base codificada de conocimientos sobre la enseñanza (Zeichner, 1993).

\section{Referencias bibliográficas}

Acker, Sandra. Género y educación. Reflexiones sociológicas sobre mujeres, enseñanza y feminismo. Madrid, España: Narcea, 1995.

Apple, Michael. Maestros y Textos. Una economía política de las relaciones de clase y de sexo en educación. Barcelona Buenos Aires - México: Paidós, 1997.

Aristóteles. Ética Nicomaquea y Política. Revisión española e introducción de Antonio Gómez Robledo. México: Porrúa, 1967.

Arnot, Madelaine. ¿La época del igualitarismo? La política y las prácticas feministas contemporáneas en educación en el Reino Unido. En Ballarin, P. (ed.). Desde las mujeres. Modelos educativos: Coeducar/Segregar. Granada, España: Universidad de Granada, 1992.

Belausteguigoitia, Marisa, Mingo, Aracelly. Fuga a dos voces. Ritmos, 
contrapuntos y superposiciones del campo de los estudios de género $\mathrm{y}$ educación. En Belausteguigoitia, Marisa, Mingo, Aracelly. Géneros prófugos. Feminismo y educación. México - Buenos Aires - Barcelona: Paidós, 1999.

Berstein, Basil. Hacia una teoría de las transmisiones educativas. Madrid, España: Akal, 1988.

Bonal, Xavier. Las actitudes del profesorado ante la coeducación. Propuesta de intervención. Barcelona, España: GRAÓ, 1997.

Bourdieu, Pierre. La distinción. Criterio y bases sociales del gusto. Madrid, España: Taurus, 1984.

Carr, Wilflred. Una teoría para la educación. Hacia una investigación educativa crítica. Madrid, España: Morata, 1996.

Cornejo, José. "Profesores que se inician en la docencia: algunas reflexiones al respecto desde América Latina" Revista Iberoamericana de Educación, (19) Pág. 51-100, 1999.

Diótima. Traer el mundo al mundo. Objeto y objetividad a la luz de la diferencia sexual. Barcelona, España: ICARIA, 1996.

Ellsworth, Elizabeth. ¿Por qué esto no parece empoderante? En Belausteguigoitia, Marisa, Mingo, Aracelly. Géneros prófugos. Feminismo y educación. México - Buenos Aires - Barcelona: Paidós, 1999.

Fenstermacher, Gary. Tres aspectos de la filosofía de la investigación sobre la enseñanza. En Wittrock, Merlin. La Investigación de la Enseñanza, I.
Enfoques, teorías y métodos. Barcelona, España. Ediciones Paidós, 1990.

Ferry, Gilles. El trayecto de la formación. Los enseñantes entre la teoría y la práctica. Buenos Aires, Barcelona, México: Paidós, 1990.

Ferry, Gilles. Pedagogía de la Formación. Buenos Aires, Argentina: Novedades Educativas, 1997.

Filloux, Jean Claude. Intersubjetividad y formación. Buenos Aires, Argentina: Novedades Educativas, 1996.

Fischel, Astrid. "Estado liberal y discriminación sexista" en Costa Rica. Revista de Ciencias Sociales (65) Pág. 2537, 1994.

García, Juan, Canal, Pedro. “¿Cómo enseñar? Hacia una definición de las estrategias de enseñanza por investigación”. Revista Investigación en la Escuela (25) Pág. 5-16, 1995.

Gimeno, José. Profesionalización docente, currículo y renovación pedagógica. Sevilla, España: Universidad de Sevilla, 1989.

Gimeno, José, Pérez, Ángel. Comprender y transformar la enseñanza. Madrid, España: Morata, 1997.

Giroux, Henry. “Teorías de la reproducción y la resistencia en la nueva sociología de la educación: un análisis crítico". Cuadernos Políticos (44) Pág. 36-65, 1985.

Gore, Jenifer. Controversias entre las pedagogías. Discursos críticos y feministas como regímenes de verdad. Madrid, España. Morata, 1996.

Hernández, Fernando, Sancho, Juana. Para enseñar no basta con saber la 
asignatura. Barcelona, Buenos Aires, México: Paidós, 1993.

Lather, Patricia. ¿Seguir en la estupidez? Resistencia estudiantil al currículum liberador. En Belausteguigoitia, Marisa, Mingo, Aracely. Géneros prófugos. Feminismo y educación. México Buenos Aires - Barcelona: Paidós. (1999).

León, Magdalena. El empoderamiento en la teoría y la práctica del feminismo. En León, Magdalena (comp.). Poder y empoderamiento de las mujeres, Bogotá, Colombia: Tercer Mundo S. A., 1997.

Librería de mujeres de Milán. No creas tener derechos. España: AURYN, 1987. Traducción (1991) de María Cinta Montagut Sancho.

Marcelo, Carlos. El pensamiento del profesor. Barcelona, España: CEAC, 1987.

McLaren, Peter. Pedagogía crítica y cultura depredadora. Barcelona, Buenos Aires, México: Paidós, 1997.

Mette, Anne. ¿Cómo pueden ayudar a las chicas las experiencias escolares? ¿Pueden ser las instituciones para un solo sexo y las agrupaciones dentro de escuelas mixtas parte de una solución? En Ballarin, P. (ed.). Desde las mujeres. Modelos educativos: Coeducar/Segregar. Granada, España: Universidad de Granada, 1992.

Orner, Mimi. Interrumpiendo los llamados para una voz de el y la estudiante en la educación liberadora. En Belausteguigoitia, Marisa, Mingo, Aracelly. Géneros prófugos. Feminismo y educación. México - Buenos Aires - Barcelona: Paidós, 1999.
Piussi, Ana. Estrellas, planetas, galaxia, infinito. En Diótima. Traer al mundo el mundo. Objeto y objetividad a la luz de la diferencia sexual. Barcelona - España: ICARIA, 1996.

Piussi, Ana. La pedagogía de la diferencia sexual. Nuevas perspectivas en Italia. En Belausteguigoitia, Marisa, Mingo, Aracelly. Géneros prófugos. Feminismo y educación. México Buenos Aires - Barcelona: Paidós, 1999a.

Piussi, Ana. Más allá de la igualdad. En Lomas, Carlos (comp.). ¿Iguales o diferentes? Género, diferencia sexual, lenguaje y educación. Méxicon - Barcelona - España: Paidós, (1999b).

Postic, Marcel. Observación y Formación de los profesores. Madrid, España: Morata, 1996.

Sobrino, Encarnación. Las ideologías pedagógicas. Buenos Aires, Argentina: Editorial Humanitas, 1987.

Subirats, Marina (1999). Género y escuela. En Lomas, Carlos (comp.). ¿Iguales o diferentes? Género, diferencia sexual, lenguaje y educación. Barcelona, España: Paidós.

Subirats, Marina. "Conquistar la igualdad: La coeducación hoy”. Revista Iberoamericana de Educación (6) Pág. 49-75, 1994.

Subirats, Marina. Con diferencia: las mujeres frente al reto de la autonomía. Barcelona, España: Icaria, 1998.

Tedesco, Juan Carlos. "Desafíos de las reformas educativas en América Latina”. Revista Propuesta Educativa (19) Pág. 19-23, 1998. 
Touraine, Alain. Igualdad y diversidad. Las nuevas tareas de la democracia. México: Fondo de Cultura Económica, 1998.

Tovar, Teresa. ¿Quién le pone el género a la escuela? Criterios para adoptar una política de género en la educación.
En Téllez, Francisco (ed.). Análisis de políticas: Documentos sobre problemas educacionales de América Latina. Santiago, Chile: CIDE, 1997.

Zeichner, Kenneth. El maestro como profesional reflexivo. Cuadernos de Pedagogía, (220) Pág. 44-49. 1993.

Sandra Araya Umaña Investigadora del Instituto de Investigaciones para el Mejoramiento de la Educación Costarricense de la Universidad de Costa Rica 